This item was submitted to Loughborough's Research Repository by the author.

Items in Figshare are protected by copyright, with all rights reserved, unless otherwise indicated.

\title{
EHD-driven mass transport enhancement in surface dielectric barrier discharges
}

PLEASE CITE THE PUBLISHED VERSION

http://dx.doi.org/10.1088/0963-0252/25/6/06LT01

\section{PUBLISHER}

IOP Publishing

\section{VERSION}

NA (Not Applicable or Unknown)

\section{PUBLISHER STATEMENT}

This work is made available according to the conditions of the Creative Commons Attribution-NonCommercialNoDerivatives 4.0 International (CC BY-NC-ND 4.0) licence. Full details of this licence are available at: https://creativecommons.org/licenses/by-nc-nd/4.0/

\section{LICENCE}

CC BY-NC-ND 4.0

\section{REPOSITORY RECORD}

Taglioli, Matteo, Alexander H. Shaw, Alec Wright, Benjamin Fitzpatrick, Gabriele Neretti, Paolo Seri, Carlo A. Borghi, and Felipe Iza. 2016. "Ehd-driven Mass Transport Enhancement in Surface Dielectric Barrier Discharges". Loughborough University. https://hdl.handle.net/2134/22785. 


\title{
EHD-driven mass transport enhancement in surface dielectric barrier discharges
}

\author{
M Taglioli ${ }^{1,2}$, A Shaw ${ }^{1}$, A Wright ${ }^{1}$, B FitzPatrick ${ }^{1}$, G Neretti ${ }^{2}$, P Seri $^{2}$, C A Borghi ${ }^{2}$, and F \\ Iza $^{1}$
}

${ }^{1}$ Wolfson School of Mechanical, Electrical and Manufacturing Engineering, Loughborough University, Loughborough, LE11-3TU, UK

${ }^{2}$ Department of Electrical, Electronic and Information Engineering, University of Bologna, Bologna, 40136, Italy

E-mail: f.iza@lboro.ac.uk

\begin{abstract}
Surface Dielectric Barrier Discharges (S-DBDs) have received renewed attention in recent years for their potential application in emerging biomedical, environmental and agricultural applications. In most of these applications, the plasma is not in direct contact with the substrate being treated and the transport of reactive species from the plasma to the substrate is typically assumed to be controlled by diffusion. Here, we demonstrate that generally this is not the case and that electrohydrodynamic (EHD) forces can produce jets that enhance the delivery of these species, thereby influencing the efficacy of the S-DBD device. In particular, we have studied the degradation of potassium indigotrisulfonate solutions exposed to S-DBDs generated in devices with annular electrodes of diameters varying between $10 \mathrm{~mm}$ and $50 \mathrm{~mm}$. All the devices were driven at constant linear power density (Watts per $\mathrm{cm}$ of plasma length) and although local plasma properties remained the same in all the devices, a three-fold efficacy enhancement was observed for devices of diameter $\sim 30 \mathrm{~mm}$ due to EHD effects.
\end{abstract}


Atmospheric-pressure Surface Dielectric Barrier Discharges (S-DBDs) have been widely investigated in the past two decades for airflow manipulation due to their mechanical simplicity, electrical control capability and low power consumption [1]. In these devices, momentum transfer from charged to neutral particles results in an electrohydrodynamic (EHD) body force, a phenomenon also known as ionic wind. In its simplest implementation, the ionic wind imparts momentum to the background gas in the direction parallel to the dielectric surface $[1,2]$. Electrical and geometrical variations, however, can also produce fluxes in other directions $[3,4]$.

In addition to flow control applications, S-DBDs have gained renewed interest in recent years for their potential use in emerging biomedical, environmental and agricultural applications, such as hand cleaning [5], preparation of plasma-activated water [6], ozone generation [7], seed treatment [8] and food preservation [9]. The plasma treatment in these systems is typically indirect and transport from the surface discharge to the sample being treated is normally assumed to be dominated by diffusion $[6,10]$.

Although all S-DBDs have the same underlying topology, namely two electrodes separated by a dielectric barrier, a number of different electrode designs, such as square [8], hexagonal [6], circular [11] and spiral [12] have been proposed in recent years. To date, however, limited attention has been paid to the influence of the electrode geometry on the efficacy of these S-DBDs, and here we report experimental results that demonstrate that the electrode pattern of the S-DBD can be used to enhance the transport of reactive species away from the surface discharge and onto the target, thereby having a significant impact on the efficacy of these devices.

S-DBDs used in this study were fabricated using a $1.5 \mathrm{~mm}$ thick glass-reinforced epoxy FR-4 substrate as the dielectric barrier, with a circular and an annular copper electrode laminated on either side (Figure 1). The ground electrode and the outer part of the high voltage annular electrode were buried in epoxy to avoid plasma formation in those areas. As a result, plasma ignites only inside the annular electrode (Figure 1). The devices were operated in ambient air, leading to the formation of a cocktail of reactive oxygen and nitrogen species $[6,10]$. To study the influence of the geometry of the electrode on the transport of these reactive species, 7 devices with electrode diameter $d$ varying between $10 \mathrm{~mm}$ and $50 \mathrm{~mm}$ were fabricated.

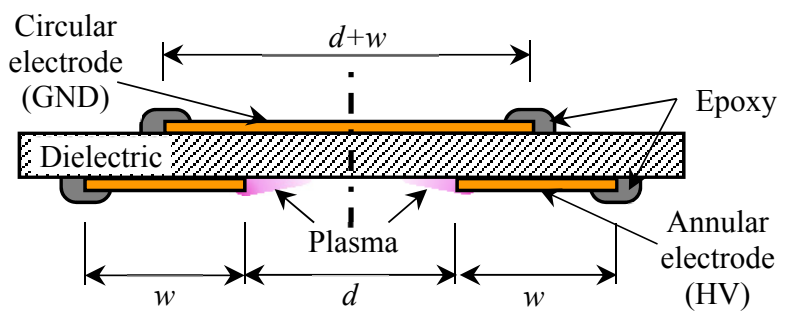

Figure 1. Schematic of the cross section of the DBD devices used in this study $(\mathrm{w}=10 \mathrm{~mm})$.

The plasmas were powered by an in-house-built resonant power supply that provided a sinusoidal voltage of 5.5 $\mathrm{kV}$ peak at a frequency of $\sim 35 \mathrm{kHz}$. Images taken using a Charge Coupled Device (CCD) camera at fixed exposure time and distance from the S-DBDs show that the plasmas extend radially $\sim 2 \mathrm{~mm}$ for all the devices. This observation agrees with existing literature in which the width of the discharge in DBD actuators is reported to be primarily controlled by the applied voltage [13], which was kept constant in all the experiments of this study. The power supply was operated both in continuous and modulated modes, and all experiments were performed in an enclosure that prevented external crossflows and reduced background light interference. 


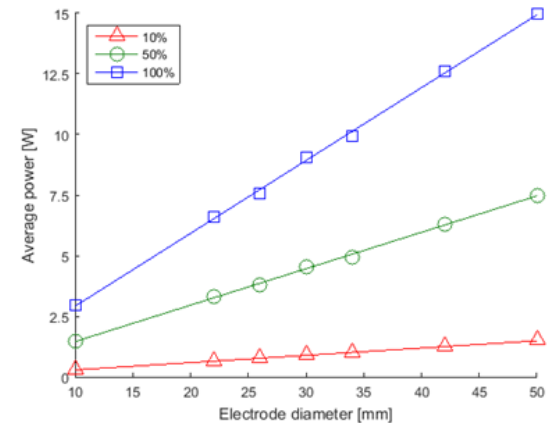

a)

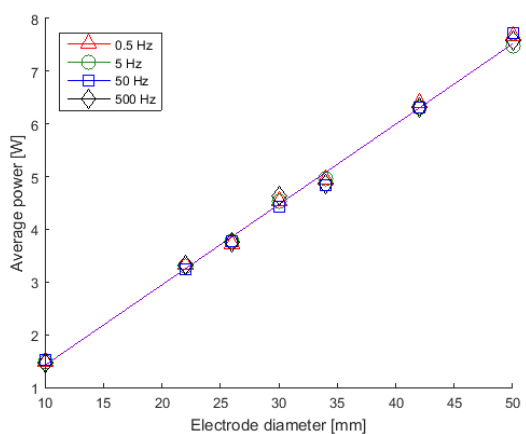

b)

Figure 2. Average power dissipated in the S-DBDs as a function of the electrode diameter $d$ a) for three different duty cycles at $5 \mathrm{~Hz}$ repetition frequency ( $100 \%$ line is continuous operation mode) and b) for different repetition frequencies at $50 \%$ duty cycle.

The time-averaged power dissipated in the plasmas was measured using charge-voltage (Q-V) Lissajous figures [14]. A $220 \mathrm{nF}$ sensing capacitor was connected in series with the plasma and the voltage across the capacitor was measured using a Tektronix P6139A voltage probe. The voltage applied across the plasma was measured using a Tektronix P6015A high-voltage probe and both traces were recorded on a Tektronix DPO4104B digital oscilloscope. Voltage signals were compensated for the different delays of the probes and the experimental error was determined to be $<5 \%$. As shown in Figure 2, the average power delivered to the plasmas increases linearly with the diameter of the device. This linear relationship indicates that the plasmas are driven at a constant linear power density (Watts per plasma length where the plasma length is $\pi d$ ), and therefore local plasma properties are expected to remain the same for all the devices when these are operated at the same duty cycle and modulation frequency.

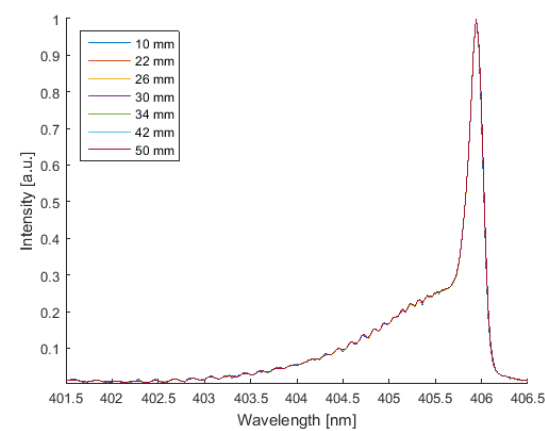

a)

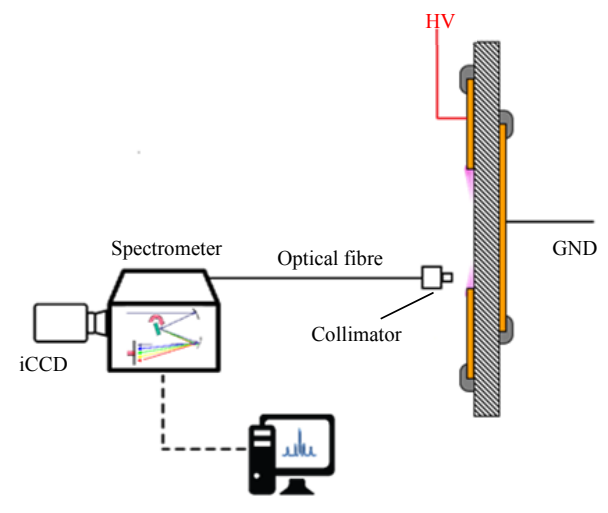

b)

Figure 3. a) Normalised spectra of the $\mathrm{N}_{2}$ SPS (0-3) ro-vibrational transition for discharges generated with S-DBDs of different diameter. Discharges sustained at $5 \mathrm{~Hz}$ modulation frequency and 50\% duty-cycle. b) Schematic of the experimental set-up.

Indeed the optical emission spectra of the plasmas generated in devices with different diameters under the same modulation frequency and duty cycle overlap with each other, indicating that the plasmas have the same chemistry and temperature. As an example, Figure 3 shows a nitrogen $\mathrm{C}^{3} \Pi_{\mathrm{u}}-\mathrm{B}^{3} \Pi_{\mathrm{g}}$ Second Positive System (SPS) band that can be used to infer the gas temperature in atmospheric plasmas $[15,16]$. The spectra were collected by means of a collimator, an Ocean Optics QR400-7-UV/VIS optical fibre and an Andor SR-303i-A Czerny-Tuner spectrograph as depicted in Figure 3b. The entrance slit and the holographic grating were set at $10 \mu \mathrm{m}$ and 2400 $1 / \mathrm{mm}$ respectively and an intensified CCD camera paired to the spectrograph was used as a detector. The camera exposure time was set to $100 \mathrm{~ms}$ and 1000 acquisitions were performed for each device. All the measured spectra were calibrated both in wavelength and intensity by means of an Ocean Optics HG-1 Mercury-Argon lamp and an Ocean Optics DH-2000-BAL Deuterium-Halogen light source respectively. The rotational temperature has been obtained by comparing the experimental spectra with a synthetic one computed using SPECAIR [17]. The rotational temperature for devices operated at $50 \%$ duty cycle (Figure $3 \mathrm{a}$ ) was found to be $360 \pm 10 \mathrm{~K}$. 
Although local plasma properties are the same for all the devices operated under the same modulation frequency and duty cycle, the EHD effect induced by devices of different dimensions can vary significantly. For circular S$\mathrm{DBD}$, the ionic wind pushes the gas radially towards the centre of the device, creating opposite flows that when meeting at the centre of the device form a jet perpendicular to the surface of the device [4]. Figure 4 shows Schlieren images of such jets formed by a $14 \mathrm{~mm}, 30 \mathrm{~mm}$ and $46 \mathrm{~mm}$ diameter S-DBDs operated at the same voltage and duty cycle. The Schlieren images were taken using the Z-type Schlieren configuration reported in [18]. Natural convection is expected to be negligible under the presence of these EHD induced jets.

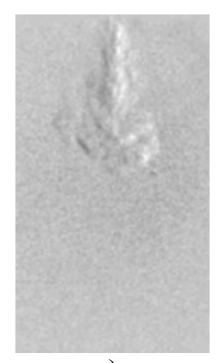

a)

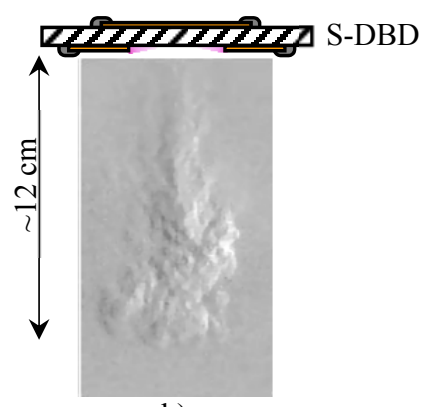

b)

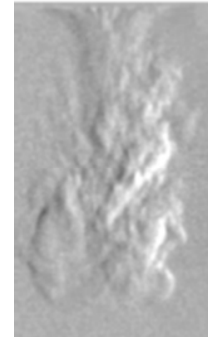

c)

Figure 4. Schlieren images of jets formed by S-DBDs: a) $d=14 \mathrm{~mm}$; b) $d=30 \mathrm{~mm}$; c) $d=46 \mathrm{~mm}$. Images recorded $40 \mathrm{~ms}$ after plasma ignition.

As shown in Figure 4, the EHD effect causes the formation of a jet perpendicular to the dielectric surface and the strength of this jet depends on the device geometry. Small devices impart little momentum to the background gas, resulting in small and weak jets. As the diameter of the device increases, so does the length and strength of the jet (compare Figure $4 \mathrm{a}$ and Figure $4 \mathrm{~b})$. Increasing the diameter beyond some critical diameter $(\sim 30 \mathrm{~mm})$, no significant increase in the length of the jet is observed. To assess the influence of these jets on the efficacy of the treatment of the S-DBDs, the degradation rate of potassium indigotrisulfonate dye (Sigma-Aldrich) solutions placed underneath S-DBDs of different diameters was measured. Changes in the degradation rate of these indigo solutions would reflect variations in the flux of reactive species delivered by the plasma to the liquid.

A volume of $150 \mathrm{ml}$ of a $2 \mu \mathrm{M}$ indigo solution was poured into a $45 \mathrm{~mm}$ high PVC cylindrical container with inner diameter of $72 \mathrm{~mm}$. Two Ocean Optics 74-UV/VIS collimating lenses were mounted facing each other on the walls of the container to transmit and receive light emitted by an Ocean Optics HL-2000-FHSA TungstenHalogen lamp. The indigo solution container was placed coaxially with the S-DBD devices at a fixed distance of $5 \mathrm{~mm}$. The time evolution of the light intensity at $605 \mathrm{~nm}$ was measured by means of an Ocean Optics QP600-1UV-VIS optical fibre and an Ocean Optics USB2000+ spectrometer. The light detected was assumed to follow the Beer-Lambert law with a potassium indigotrisulfonate molar absorbance of $\varepsilon=23800 \mathrm{M}^{-1} \cdot \mathrm{cm}^{-1}$ at $605 \mathrm{~nm}$ [19]. The degradation rate was determined as the slope of the time evolution of the indigo concentration. Experiments were performed in triplicate and the standard deviation between experiments was $<6 \%$. Results are shown in Figure 5.

Based on the data presented above and neglecting EHD effects, one would expect that for a fixed repetition frequency and duty cycle the degradation rate of the indigo solutions would increase linearly with the diameter of the S-DBD. This linear increase would be attributed to the longer plasmas produced in bigger devices (plasma length $=\pi d$ ). As shown in Figure 5, however, the dependence of the degradation rate of the indigo solution on the device diameter is not linear and an enhanced performance ( $\sim$ three fold) is observed for devices with a diameter of $\sim 30 \mathrm{~mm}$. To highlight this, dash-dotted lines indicating what the linear dependence would look like at various duty cycles has been introduced in Figure 5a. This enhancement above the expected linear increase is attributed to EHD effects, in particular, the formation of a jet that enhances the transport of reactive species from the plasma to the liquid (Figure 4). Although the degradation rate depends both on the duty cycle and the repetition frequency used to generate the plasmas (Figure 5), the optimum device diameter in all cases studied here is found to be $\sim 30 \mathrm{~mm}$. 


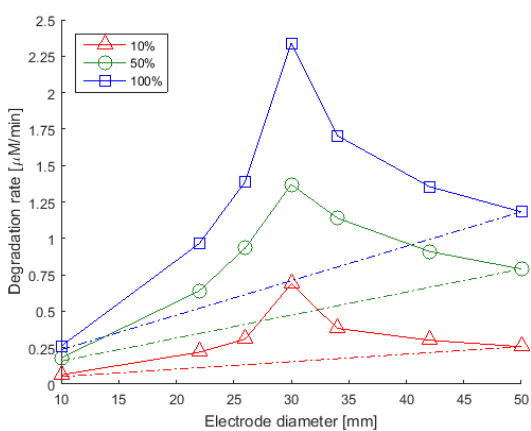

a)

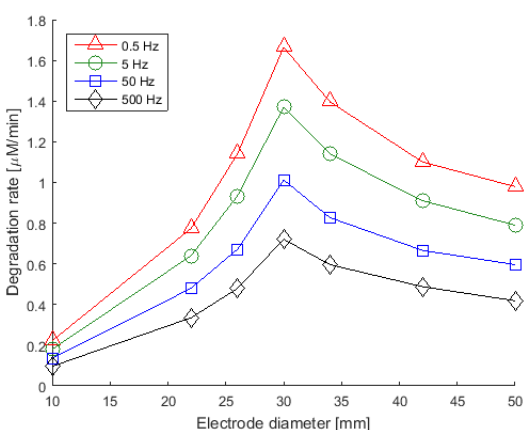

b)

Figure 5. Degradation rate of $2 \mu \mathrm{M}$ potassium indigotrisulfonate solutions as a function of the electrode diameter of the S-DBD. Discharges sustained a) at various duty cycles with a modulation frequency of $5 \mathrm{~Hz}$. Dash-dotted lines represent linear increases that can be attributed to the longer plasma formed in larger devices. b) at $50 \%$ duty cycle for various repetition frequencies.

The enhanced degradation rate observed with devices of $30 \mathrm{~mm}$ diameter implies that an increased flux $(\Gamma)$ of reactive species reaches the indigo solution. Since $\Gamma=c \cdot v$ where $c$ is the concentration of reactive species and $v$ their velocity component perpendicular to the liquid surface, an increase in flux could be caused by an increase in the concentration of reactive species $(c)$ and/or an increase in the gas velocity $(v)$. To gain further insights into the underlying mechanism causing the enhanced degradation, we performed an experiment in which the ozone concentration produced by the S-DBDs was measured under different driving conditions (Figure 6). Ozone was chosen because it can be readily measured non-intrusively by optical means and it is one of the key reactive species causing the degradation of the indigo solution $[19,20]$. The measurements were performed using two fibres (Ocean Optics QP600-1-UV-VIS) separated by $72 \mathrm{~mm}$. The fibres faced each other and they were terminated with two Ocean Optics 74-UV/VIS collimating lenses. An Ocean Optics LLS-255 lamp was used as a source of UV radiation and the transmitted light was recorded with an Ocean Optics HR2000+ high resolution spectrometer. The time evolution of the ozone concentration was computed assuming that the absorption of photons by $\mathrm{O}_{3}$ molecules follows the Beer-Lambert law with an absorption cross section at $254 \mathrm{~nm}$ of $\sigma=1.12 \cdot 10^{-}$ ${ }^{17} \mathrm{~cm}^{2}$ [21]. Experiments were performed five times and the standard deviation of the measurements was less than $10 \%$. Since devices driven at the same modulation frequency and duty cycle operate at constant linear power density (Figure 2), the chemistry per unit length produced in the discharges is expected to be the same. In other words, in the absence of non-local effects in the afterglow, the ozone concentration is expected to increase linearly with the device diameter for devices driven under the same conditions.

The measured average ozone concentration was found to increase linearly with the electrode diameter (Figure 6) for discharges operated at the same modulation frequency and duty cycle, and importantly no deviation from this linear behaviour was observed in devices of $\sim 30 \mathrm{~mm}$ diameter. It is noted that the input power used in these experiments was low enough as to avoid a mode transition during the experiment [22]. Since the duty cycle has a direct influence on the power delivered to the plasma (Figure 2), the ozone concentration also depends on the duty cycle. On the other hand, for the conditions explored in this work, no difference in ozone concentration is observed for plasmas driven at different modulation frequencies but the same duty cycle, i.e. under the same input power conditions (Figure 6b).

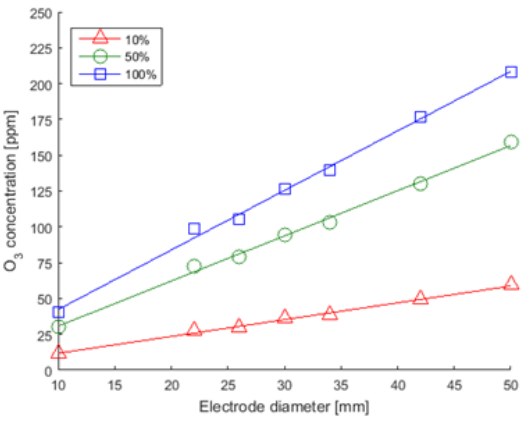

a)

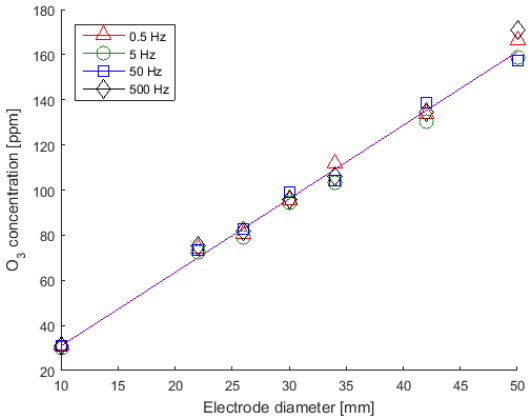

b)

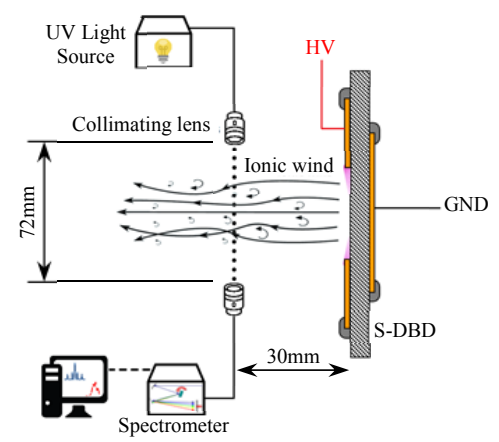

c) 
Figure 6. Ozone concentration measured 30mm away from the S-DBD. Discharges sustained a) at various duty cycles with a modulation frequency of $5 \mathrm{~Hz}$ and b) at $50 \%$ duty cycle for various repetition frequencies. c) Experimental set-up for the ozone concentration measurements.

Therefore it is concluded that the enhanced degradation of the indigo solutions observed with devices of $30 \mathrm{~mm}$ diameter is due to an increased delivery velocity of the reactive species rather than to an increase in their concentration. This enhanced velocity is caused by the formation of a jet by EHD forces, which happens to be most effective in devices of $\sim 30 \mathrm{~mm}$. It is interesting to note that even though changing the modulation frequency between $0.5 \mathrm{~Hz}$ to $500 \mathrm{~Hz}$ does not affect the input power (Figure 2b), gas temperature (data not shown explicitly) and ozone concentration (Figure $6 \mathrm{~b}$ ), the repetition frequency influences the efficacy of the device in degrading the indigo solution (Figure 5b). This dependence on the repetition frequency is a further indication of the importance of EHD forces as it is well known that the momentum coupling to the background gas is less effective at increasing modulation frequencies [23]. The decreasing performance for devices larger than 30mm (Figure 5) suggests that the velocity of the jet peaks at $30 \mathrm{~mm}$ and decreases in larger devices. This decrease could be caused by an increased turbulence as suggested by images in Figure 4. Experiments are on the way to test this hypothesis and results will be reported elsewhere.

In summary, surface dielectric barrier discharges have attracted renewed interest for emerging applications in the biomedical, environmental and food sectors. Transport of reactive species from the plasma to the samples being treated is critical in these emerging applications. This transport is typically assumed to be diffusion controlled but in this letter it is demonstrated that the transport can be influenced by electrohydrodynamic (EHD) forces and therefore electrodes can be engineered to enhance this transport. For the circular S-DBD discharges studied in this work, the enhanced transport caused by the formation of a jet perpendicular to the surface of the device causes the efficacy of the S-DBD in degrading an indigo solution to increase by a factor of 3 from what would otherwise be expected from power and plasma size considerations. Similar effects are expected in S-DBD with other electrode geometries (e.g. square and hexagonal patterns) and further research should address not only the optimization of one discharge but also the interaction between adjacent discharges.

\section{References}

\footnotetext{
${ }^{1}$ Moreau E 2007 Journal of Physics D: Applied Physics 40605

${ }^{2}$ Neretti G, Cristofolini A, Borghi C A, Gurioli A and Pertile R 2012 IEE Transactions on Plasma Science 401678

${ }^{3}$ Santhanakrishnan A and Jacob J D 2007 Journal of Physics D: Applied Physics 40637

${ }^{4}$ Neretti , Cristofolini A and Borghi C A 2014 Journal of Applied Physics 115163304

${ }^{5}$ Morfill G E, Shimizu T, Steffes B and Schmidt H U 2009 New Journal of Physics 11115019

${ }^{6}$ Liu D X, Liu Z C, Chen C, Yang A J, Li D, Rong M Z, Chen H L and Kong M G 2016 Scientific Reports 623737

${ }^{7}$ Williamson J M, Trump D D, Bletzinger P and Ganguly B N 2006 J. Phys. D: Appl. Phys. 394400

${ }^{8}$ Mitra A, Li Y-F, Klämpfl T G, Shimizu T, Jeon J, Morfill G E and Zimmermann J L 2014 Food Bioprocess Technol 7645

${ }^{9}$ Shaw A, Shama G and Iza F 2015 Biointerphases 1029402

${ }^{10}$ Sakiyama Y, Graves D B, Chang H-W, Shimizu T and Morfill G E 2012 J. Phys. D: Appl. Phys. 45425201

${ }^{11}$ Shaw A, Seri P, Borghi C A, Shama G and Iza F 2015 J. Phys. D: Appl. Phys. 48484001

12 Potts H E, Diver D A, Everest P C and O'Connor R D 2011 Proceedings of the 30 ${ }^{\text {th }}$ International Conference on Phenomena in Ionized Gases (ICPIG), Belfast, 296

${ }^{13}$ Enloe C L, McLaughlin T, Van Dyken R, Kachner K, Jumper E and Corke T C 2004 AIAA Journal 42589

${ }^{14}$ Manley T C 1943 Journal of The Electrochemical Society 8483

15 Bruggeman P J, Sadeghi N, Schram D C and Linss V 2014 Plasma Sources Sci. Technol. 23023001

${ }^{16}$ Iza F and Hopwood J A 2004 IEEE Trans. Plasma Sci. 32498

${ }^{17}$ Laux C O 2008 Optical Diagnostics and Collisional-Radiative Models, Von Karman Institute Course on Hypersonic and Cruise Vehicles, Stanford University

${ }^{18}$ Cristofolini A, Neretti G, Roveda F and Borghi C A 2012 J Appl Phys 111033302

${ }^{19}$ Bader H and Hoigné J 1981 Water Research 15449

${ }^{20}$ Bruggeman P et al 2016 Plasma Sources Science and Technology (accepted)

${ }^{21}$ Grebenshchikov S Y, Qu Z W, Zhu H and Schinke R 2007 Phys. Chem. Chem. Phys. 92044

${ }^{22}$ Shimizu T, Sakiyama Y, Graves D B, Zimmermann J L and Morfill G E 2012 New J. Phys. 14103028

${ }^{23}$ Glezer A, Amitay M and Honohan A M 2005 AIAA Journal 431501
} 